Focused ion beam creation and templating of $\operatorname{In} A s$ and $\operatorname{InAs} / \ln P$ nanospikes

This article has been downloaded from IOPscience. Please scroll down to see the full text article.

2011 Nanotechnology 22355302

(http://iopscience.iop.org/0957-4484/22/35/355302)

View the table of contents for this issue, or go to the journal homepage for more

Download details:

IP Address: 141.211.173.82

The article was downloaded on 06/04/2012 at 16:20

Please note that terms and conditions apply. 


\title{
Focused ion beam creation and templating of InAs and InAs/InP nanospikes
}

\author{
K A Grossklaus and J M Millunchick \\ Department of Materials Science and Engineering, University of Michigan, Ann Arbor, \\ MI 48109-2136, USA \\ E-mail: joannamm@umich.edu
}

Received 29 April 2011, in final form 15 July 2011

Published 5 August 2011

Online at stacks.iop.org/Nano/22/355302

\begin{abstract}
Ion beam irradiation has been examined as a method for creating nanoscale semiconductor pillar and cone structures, but has the drawback of inaccurate nanostructure placement. We report on a method for creating and templating nanoscale InAs spikes by focused ion beam (FIB) irradiation of both homoepitaxial InAs films and heteroepitaxial InAs on InP substrates. These 'nanospikes' are created as In droplets, formed due to FIB irradiation, act as etch masks for the underlying InAs. By pre-patterning the InAs to influence In droplet movement, nanospike locations on homoepitaxial InAs may be controlled with limited accuracy. Creating nanospikes using an InAs/InP heterostructure provides an additional measure of control over where the spikes form because nanospikes will not form on exposed regions of InP. This effect may be exploited to accurately control nanospike placement by pre-patterning an InAs/InP heterostructure to control the location of the InAs/InP interface. Using this heterostructure templating method it is possible to accurately place nanospikes into regular arrays that may be useful for a variety of applications.
\end{abstract}

\section{Introduction}

The creation of III-V compound semiconductor nanostructures, and in particular high aspect ratio nanostructures such as nanowires, has become an area of intense study. The small size and unique transport properties of these structures make them promising for use in nanoscale electronic, optoelectronic, photonic [1-3], and thermoelectric applications [4, 5]. Broad beam ion irradiation of III-V semiconductor surfaces has previously been demonstrated as a method for creating high aspect ratio cone and pillar nanostructures in a variety of compound semiconductors, such as GaAs [6], InP [7-9], and $\mathrm{GaSb}[10,11]$. The structures reported in the literature have been created using homogeneous material, and heterostructures have yet to be demonstrated using a similar ion beam method. These ion beam based approaches allow for the creation of nanostructures over a wide area, but do not allow for careful control of their placement. Both the creation of heterostructures and accurate nanostructure placement will be needed in order for ion beam created structures to be useful for many nanotechnology applications.

In this work we examine the creation and templating of nanoscale spikes by normal incidence focused ion beam
(FIB) sputtering of homoepitaxial InAs and heteroepitaxial InAs/InP. The creation of group III rich droplets or nanodots by FIB irradiation of InAs [12, 13] and InP [14] is a well documented phenomenon, but the creation of high aspect ratio spike structures by FIB has not previously been reported for either material. For nanospikes created by irradiation of homoepitaxial InAs, we have found that nanospike density is controlled by the FIB irradiation parameters and the local morphology of the InAs surface, with the density of the nanospikes varying greatly due to the ongoing creation of additional nanospikes throughout the irradiation process. By FIB pre-patterning InAs before irradiation it was found that the locations of nanospikes may be partially controlled. However, the accuracy of templating using homoepitaxial InAs was limited and did not persist with extended ion erosion. For nanospikes created by irradiation of InAs/InP heterostructures, nanospike density and placement are also controlled by the FIB irradiation parameters and local InAs film morphology. However, the recession of the InAs/InP interface and exposure of the InP substrate also influence their location because nanospikes are unable to form on InP. Nanospike height and density are influenced by initial InAs film thickness, as nanospike growth is limited to those areas where InAs is 
still present. Thus, the use of an InAs/InP heterostructure provides another means by which nanospike formation can be controlled. Using film pre-patterning to template and control the InAs/InP interface location followed by ion beam exposure, it was found that nanospikes could be created accurately in regular arrays. This hybrid pre-patterning and FIB exposure method allows for the creation of nanospikes at controlled locations, thus providing some of the benefits of both serial and self-organized nanostructure creation processes.

\section{Experimental details}

Nanospikes were produced by growing InAs films on $\langle 001\rangle$ oriented InAs and InP substrates using molecular beam epitaxy (MBE) and then exposing the films to FIB irradiation. $500 \mathrm{~nm}$ thick homoepitaxial InAs films and 200 and $500 \mathrm{~nm}$ thick InAs films on InP were grown at temperatures between $460{ }^{\circ} \mathrm{C}<$ $T<470{ }^{\circ} \mathrm{C}$ at a rate of $0.2-0.35 \mathrm{ML} \mathrm{s}^{-1}$ under an $\mathrm{As}_{4}$ overpressure. For the InAs/InP case, the growth temperature was varied in order to control film roughness, with samples grown at $460^{\circ} \mathrm{C}$ being significantly rougher than those grown at $470{ }^{\circ} \mathrm{C}$. Following growth, the samples were placed in a FEI Nova Nanolab FIB/SEM system and irradiated using a normal incidence, $30 \mathrm{keV} \mathrm{Ga}^{+}$ion beam. FIB exposure was carried out using beam currents varying from $7 \mathrm{pA}$ to $7 \mathrm{nA}$ and spot dwell times varying from $100 \mathrm{~ns}$ to $10 \mu \mathrm{s}$. The FIB was scanned in a serpentine pattern over $5 \mu \mathrm{m} \times 5 \mu \mathrm{m}$ areas repeatedly using a $50 \%$ spot overlap. FIB parameters were varied in order to determine the optimum conditions for nanospike creation. For templating experiments, arrays of square mesas were first pre-patterned by inputting the mesa array as a bitmap into the FIB control software and then scanning a $30 \mathrm{kV}, 0.1 \mathrm{nA}$ FIB to match the image. Spot dwell times were varied from 10 to $50 \mu \mathrm{s}$ and the number of beam passes was varied to mill array patterns of different depths. Nanospikes were then created by irradiating the mesa arrays using the method described above.

Characterization of the nanospikes was carried out during and after their creation by scanning electron microscopy (SEM). Nanospikes created using InAs/InP heterostructures were also examined by transmission electron microscopy (TEM). During irradiation the FIB was blanked at regular intervals to allow SEM imaging of different points in the nanospike formation process. Cross-sectional samples for TEM analysis were created using a modified H-bar method [15], during which an InAs/InP sample was first thinned by FIB to $<5 \mu \mathrm{m}$ followed by nanospike creation on the thinned region. All sample thinning was carried out before nanospike creation in order to prevent redeposition of sputtered material in the regions containing nanospikes. Nanospikes created in this manner projected above the thicker region, allowing them to be viewed in transmission without the need for any additional FIB thinning following their production. Diffraction contrast and high resolution TEM imaging of the nanospikes were carried out using both a JEOL 3011 TEM and a JEOL 2010F TEM. High angle annular dark-field scanning transmission electron microscopy (HAADF STEM) imaging and STEM energy-dispersive spectrometry (EDS) composition analysis of the InAs/InP nanospikes were conducted using the JEOL 2010F system.

\section{Results and discussion}

\subsection{Nanospike creation}

Nanospikes form as the homoepitaxial InAs film and underlying wafer are eroded by successive passes of the ion beam (figure 1). Shortly after the start of FIB irradiation, In droplets form on the InAs film due to preferential sputtering of As $[12,16]$ (figure 1(a)) and then migrate randomly across its surface. With continued FIB erosion and increasing amounts of excess In present the film forms ripples that result in a raised, web-like pattern of In globules and recessed areas (figure 1(b)). This rippling transition may be caused by changes in sputter yield due to variation in local curvature [17] and due to local shadowing from the presence of excess surface In. Some of the indium droplets traveling across the uneven eroded surface eventually come to apexes in the ripple pattern, where they become stationary. As a result the droplets mask the underlying material, causing nanospikes to form and continue to grow at these locations as the surrounding material is milled away (figure 1(b)). As erosion proceeds the rippled morphology persists and additional nanospikes form as In droplets come to other apexes at later times. This process of continuous nanospike formation with ion erosion results in a random distribution of InAs nanospikes with a broad range of heights (figure 1(c)). The formation and placement of the nanospikes depend strongly on the irradiation parameters and the morphology of the ion-eroded InAs. Low beam currents and short spot dwell times were found to aid nanospike creation. No nanospikes were produced using beam currents greater than $\sim 50 \mathrm{pA}$ or dwell times longer than $1 \mu \mathrm{s}$. The tallest and greatest number of nanospikes was produced using an approximately $7 \mathrm{pA}$ beam and $100 \mathrm{~ns}$ dwell time. Using these optimum beam parameters and 12000 passes of the ion beam, nanospikes with a density of $2.84 \pm 0.8$ spikes $\mu \mathrm{m}^{-2}$ could be produced from homoepitaxial InAs. The final nanospike height depends on how early in the erosion process nanospikes begin to form and the total number of ion beam passes. Using 12000 beam passes, nanospikes were produced in a wide range of heights with an average of $400 \pm 200 \mathrm{~nm}$ and an average diameter measured at half maximum height of $120 \pm 25 \mathrm{~nm}$. The tallest nanospike created using homoepitaxial InAs and 12000 beam passes was $\sim 975 \mathrm{~nm}$. Increasing the number of beam passes causes more nanospikes to form and causes the existing nanospikes to increase in height.

Nanospikes that form by FIB irradiation of an InAs/InP heterostructure do so in a manner similar to the homoepitaxial InAs case, but with some important differences. Figure 2(a) shows a rough, faceted, $500 \mathrm{~nm}$ thick InAs film on InP before irradiation. As in the homoepitaxial InAs case, In droplets form on the InAs film due to preferential sputtering and with continued FIB exposure the InAs film forms a raised, weblike pattern (figure 2(b)). As the underlying InP becomes partially exposed, nanospikes form and increase in height at locations where large In droplets become stationary on the web-like pattern (figure 2(c)). Once the InAs film has been completely milled away the change in nanospike height slows, and nanospike width and height eventually begin to 

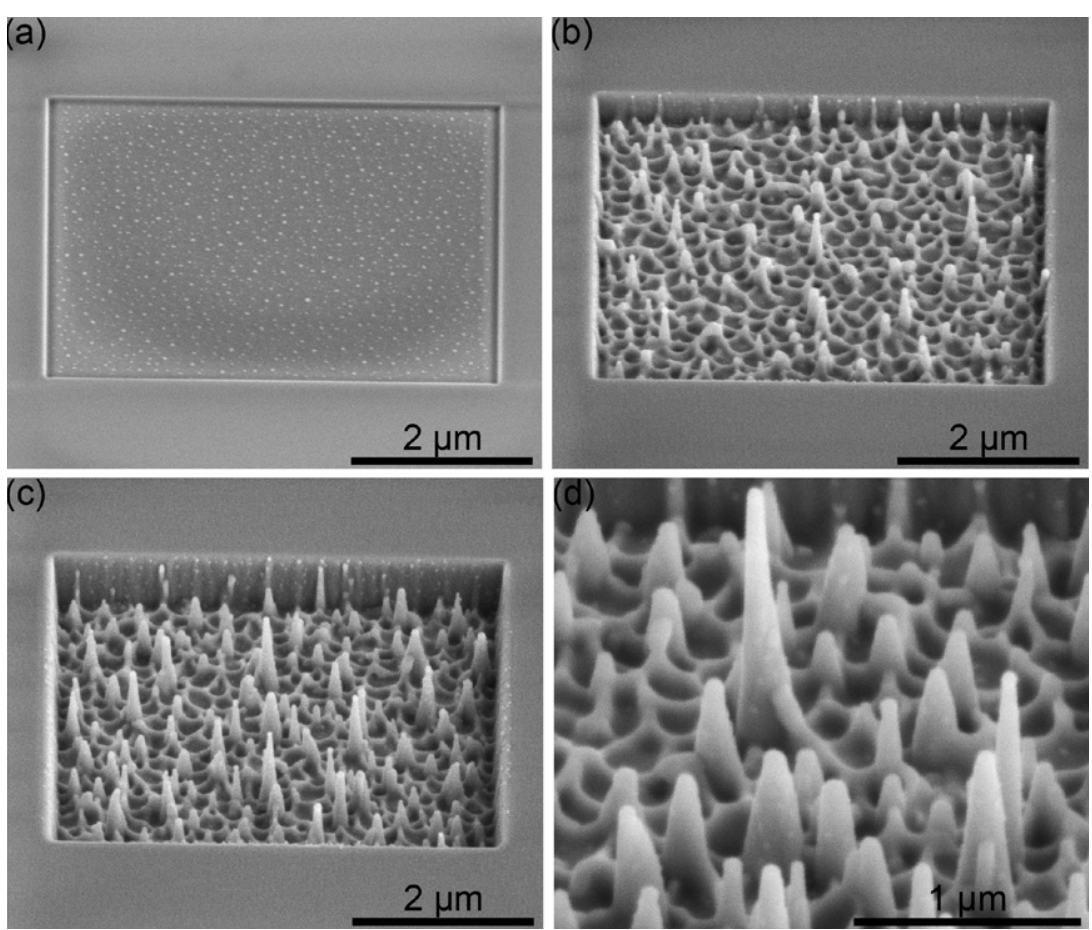

Figure 1. SEM images showing a region of homoepitaxial InAs through the process of irradiation with a $30 \mathrm{kV} 7.3 \mathrm{pA}$ ion beam using a $100 \mathrm{~ns}$ spot dwell time. (a) shows the region after 1000 beam passes, with small In droplets present. (b) shows the area following 7000 beam passes, with a few nanospikes visible. (c) shows the area after 12000 beam passes, with many nanospikes visible. (d) shows a higher magnification image of some InAs nanospikes produced after 15000 beam passes. Images were taken with the sample tilted $52^{\circ}$ off the electron beam normal.
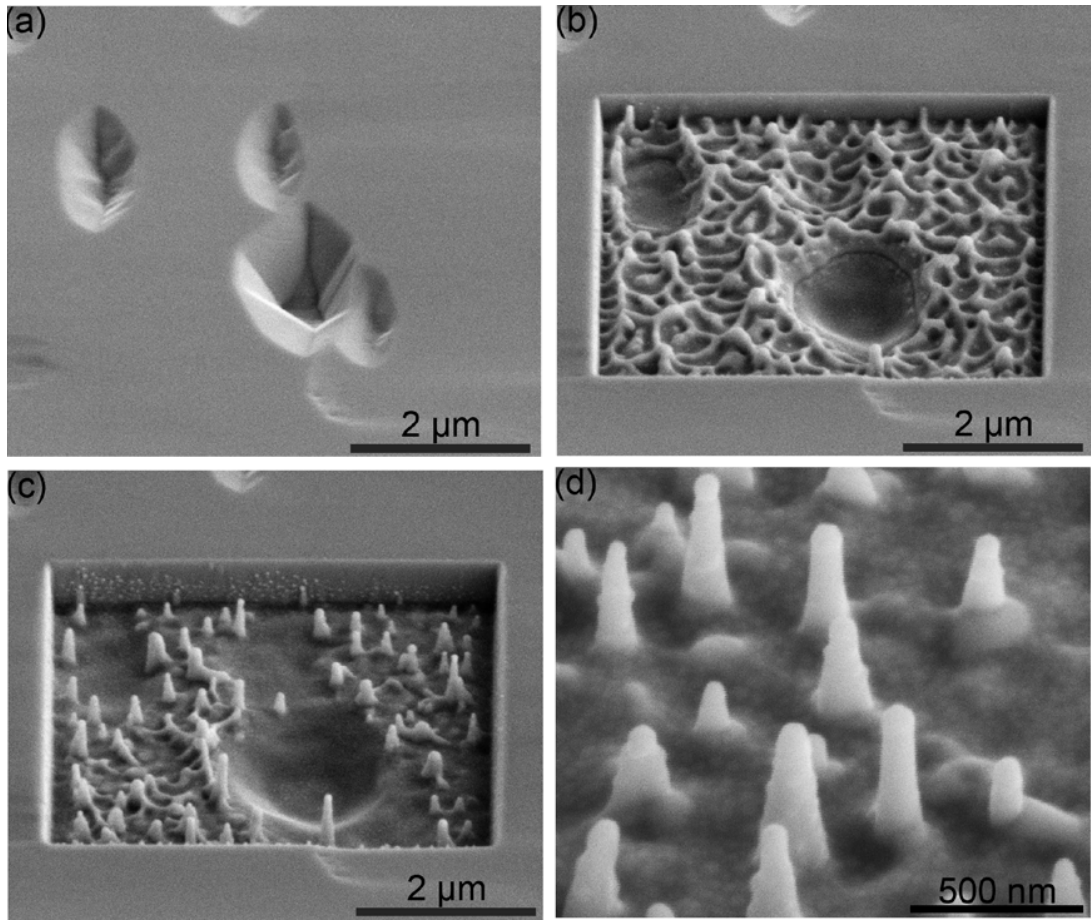

Figure 2. SEM images showing a region of partially rough InAs film grown on InP through the process of irradiation with a $30 \mathrm{kV} 7.4 \mathrm{pA}$ ion beam using a $100 \mathrm{~ns}$ spot dwell time. (a) shows the region before exposure. (b) shows the same location following 7000 beam passes, with regions of InP exposed. (c) shows the area following 12000 passes with nanospikes present. (d) shows a higher magnification view of nanospikes representative of those produced in this study using an InAs/InP heterostructure. Images were taken with the sample tilted $52^{\circ}$ off the electron beam normal. 


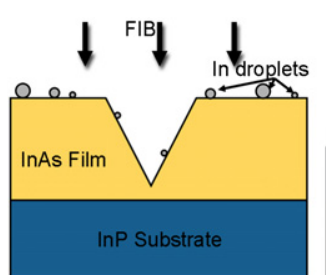

(a)

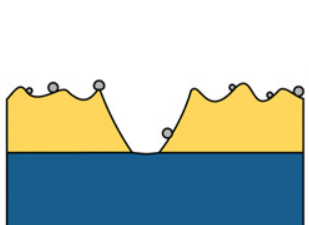

(b)

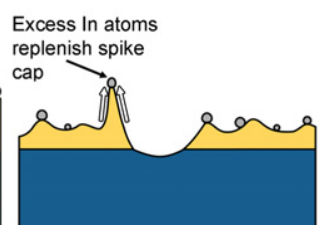

(c)

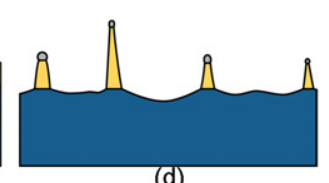

(d)

Figure 3. Schematic showing the mechanism of nanospike formation. (a) FIB irradiation causes In droplets to form on an initially rough InAs film. (b) As irradiation continues the InAs surface becomes uneven and rippled. (c) An In droplet becomes stationary at an apex of the film and will act as a protective mask for the underlying InAs, allowing a nanospike to form. The mask is replenished by excess In produced as the surrounding InAs erodes. (d) More nanospikes will form as other In droplets become stationary until the InAs film is eroded away and the underlying InP substrate is fully exposed. Nanospikes will not form on the InP regions.

(This figure is in colour only in the electronic version)

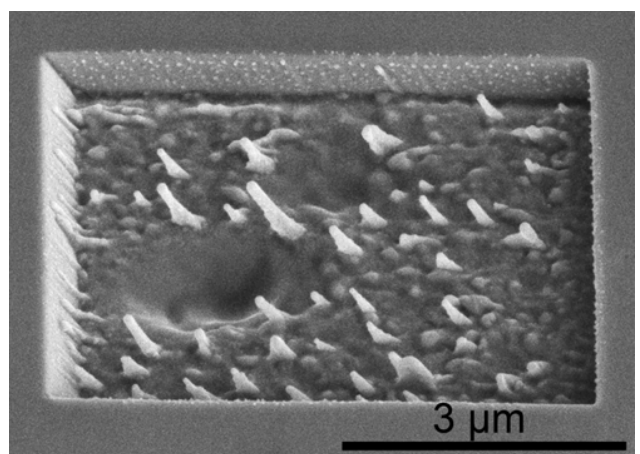

Figure 4. SEM image of nanospikes produced by tilting an InAs/InP heterostructure sample $30^{\circ}$ off normal from the FIB.

decrease. No large In droplets or nanospikes formed on the regions of exposed InP. As with the homoepitaxial film, lower beam currents and dwell times resulted in taller and more densely packed nanospikes. The tallest and greatest number of nanospikes were created using an approximately $7 \mathrm{pA}$ beam and $100 \mathrm{~ns}$ dwell time, requiring in excess of 10000 beam passes to fully erode a $500 \mathrm{~nm}$ InAs film. Using these optimum beam parameters, an average density of $1.98 \pm 0.5$ spikes $\mu \mathrm{m}^{-2}$ was produced. The final average nanospike height increases with InAs film thickness. Using a $500 \mathrm{~nm}$ InAs starting film, nanospikes were created in a wide range of heights with an average of $300 \pm 100 \mathrm{~nm}$ and an average diameter measured at half maximum height of $110 \pm 30 \mathrm{~nm}$. The maximum observed nanospike height was approximately $875 \mathrm{~nm}$, greater than the initial $500 \mathrm{~nm}$ thickness of the InAs film, demonstrating that in some cases nanospikes continue to grow taller after erosion of the InAs film is complete. This occurs because the nanospike's In droplet mask still requires a finite amount of time to be sputtered away once the surrounding InAs has been removed, and so will still protect the nanospike for a short period.

The physical mechanism of nanospike formation is similar for nanospikes produced using both homoepitaxial InAs and InAs/InP heterostructures. In both cases nanospike density and placement are determined by surface energy effects and differences in material sputtering behavior, and these properties are determined locally by InAs film morphology and the quantity of excess In present. The formation of nanospikes on an InAs/InP heterostructure deviates from the
InAs case in that the different sputtering rates of InAs and InP and the location of the InAs/InP interface also effect nanospike creation. Figures 3(a)-(c) illustrate the mechanism of nanospike formation as it pertains to both the InAs and InAs/InP cases, while figure 3(d) illustrates the role of the InAs/InP interface for the heterostructure case. With the start of FIB exposure, In droplets form on the flat InAs surface by preferential sputtering of As (figure 3(a)) and become mobile due to ion beam induced migration. During the intermediate stages of InAs film erosion, a web-like ripple pattern forms as In droplets continue to migrate across the surface. Eventually some of the droplets reach the apexes of the ripple pattern and become stationary (figure 3(b)), masking the underlying film and allowing nanospikes to form (figure 3(c)). Droplets are likely driven toward apexes in the rippled InAs film in order to reduce their contact area with the InAs and so reduce their interfacial surface energy. Tilting the sample off normal relative to the FIB resulted in nanospikes being produced tilted by the same amount, further verifying this etch masking mechanism. Figure 4 shows nanospikes created by tilting an InAs/InP heterostructure surface $30^{\circ}$ from the direction normal to the ion beam. In the case of formation on homoepitaxial InAs, nanospikes grow under stationary In droplets so long as the In droplet is maintained. The In droplet 'etch mask' is resupplied by excess indium atoms from preferential sputtering of the III-V material, preventing it from being immediately sputtered away. This group III 'selfsustained etch-mask' mechanism has previously been proposed in order to explain the formation of Ga capped cones on ion irradiated GaSb [11, 18]. For a homoepitaxial InAs film, additional nanospikes can form in the surrounding area as more In droplets become stationary and act as masks at later times. This is not the case for an InAs/InP heterostructure, because once the underlying InP is exposed the location of the InAs/InP interface and the different FIB sputtering behaviors of InAs and InP control the locations at which large In droplets can be present to form nanospikes. As the InAs/InP surface is eroded the large In droplets needed for nanospike formation are driven from the InAs/InP interface in order to remain on the InAs. This indicates that the interfacial surface energy of a droplet on InAs is likely lower than on InP. Additionally, large In droplets were only able to form and persist on the InAs. As has been previously reported, FIB sputtering of InAs is expected 

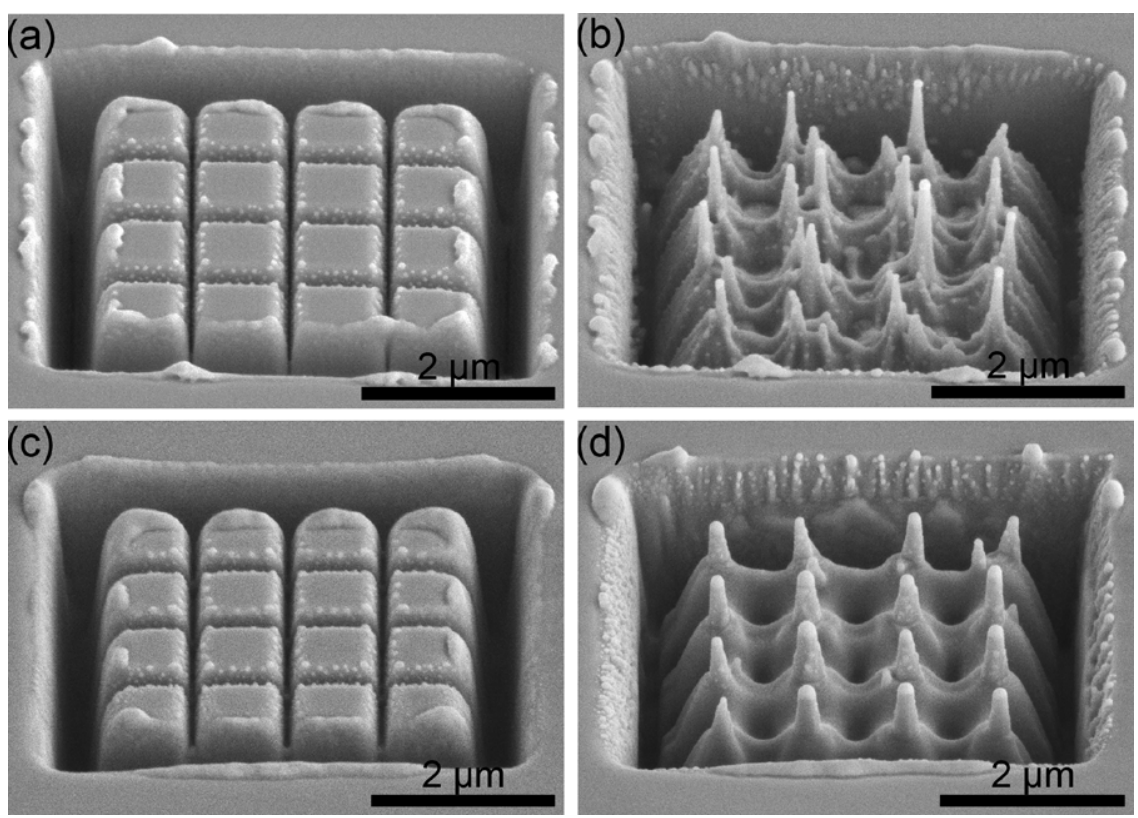

Figure 5. SEM images of templated areas of both homoepitaxial InAs and an InAs/InP heterostructure. (a) shows an array of raised mesas created by patterning homoepitaxial InAs. (b) shows that same array following 9000 ion beam passes, with InAs nanospikes visible. (c) shows an array of raised mesas created by patterning the $500 \mathrm{~nm}$ InAs/InP heterostructure. (d) shows that same InAs/InP array following 9000 ion beam passes with nanospikes visible. The mesa templates were created using similar beam parameters and for both cases the templates were irradiated using the identified optimum beam parameters for nanospike creation. In both (b) and (d) the nanospikes form at locations roughly matching the original mesa locations.

to produce more excess In atoms per ion and produce much larger In droplets than sputtering of InP [16]. Indeed, only small group III droplets $(<45 \mathrm{~nm})$ were observed on regions of exposed InP, while larger droplets $(>70 \mathrm{~nm})$ which could produce nanospikes where seen on the InAs film. This and the absence of any new nanospike formation on InP indicates that sputtering of InP alone is unable to produce or sustain In droplets large enough for nanospike formation. Thus exposure of the underlying InP substrate acts as a local inhibitor for nanospike formation, and movement of the InAs/InP interface as the InAs film erodes acts to constrain the regions in which new nanospikes can form. Due to this effect any initial film roughness causes the InAs to ripple more quickly and exposes the InP substrate sooner, thus playing a significant role in determining final nanospike location. Once the InAs film is milled through, nanospike height increase slows and eventually stops (figure $3(\mathrm{~d})$ ). This is again likely because the InP substrate etches more slowly and produces fewer excess In atoms than InAs [16], and so is incapable of resupplying the droplet etch mask faster than it is being sputtered by the ion beam. Upon exposure of the InP substrate, nanospikes may grow for a limited time while their masking droplet still protects them, but as their droplet is sputtered away they cease to grow and also begin to be sputtered away. As a result, the thickness of the InAs film present when nanospike formation starts acts to control the maximum height a nanospike can reach.

\subsection{Nanospike templating}

The mechanism for nanospike formation proposed above suggests that the locations at which nanospikes form may be controlled by templating the starting InAs surface to limit the locations where In droplets come to rest as FIB erosion proceeds. FIB pre-patterning of a homoepitaxial InAs sample was observed to partially control the locations at which nanospikes form. Figure 5(a) shows a group of $1 \mu \mathrm{m} \times$ $1 \mu \mathrm{m}$ mesas created by FIB milling an InAs sample, and figure 5(b) shows the same area following 9000 passes with a $7.3 \mathrm{pA}$ FIB beam using a $100 \mathrm{~ns}$ dwell time, resulting in the formation of nanospikes in a pattern roughly matching the original pre-patterned grid. This controlled placement is the result of both the mesa pattern limiting the area over which the In droplets can migrate and more rapid sputtering from the mesa edges acting to create an inwardly receding boundary that drives the In droplets toward the mesa interior. In the case of homoepitaxial InAs the surface in the patterned area remains rippled and new In droplets are produced as erosion continues both on the FIB patterned mesas and in the regions between them. As a result, prolonged FIB erosion causes secondary nanospike formation between the mesas of the original template, disrupting the nanospike pattern. This effect is particularly pronounced in cases where the original mesa pattern was not milled deeply. In those experiments the mesa pattern was quickly removed by FIB milling and nanospike formation was able to take place unrestricted across the pre-patterned region. Thus pre-patterning and exposure of homoepitaxial InAs allows only limited nanospike templating to occur.

Nanospike formation on pre-patterned InAs/InP heterostructures allows for more accurate and reproducible nanospike templating. Figure 5(c) shows a group of $1 \mu \mathrm{m} \times$ $1 \mu \mathrm{m}$ mesas created by FIB milling an InAs/InP sample, and 

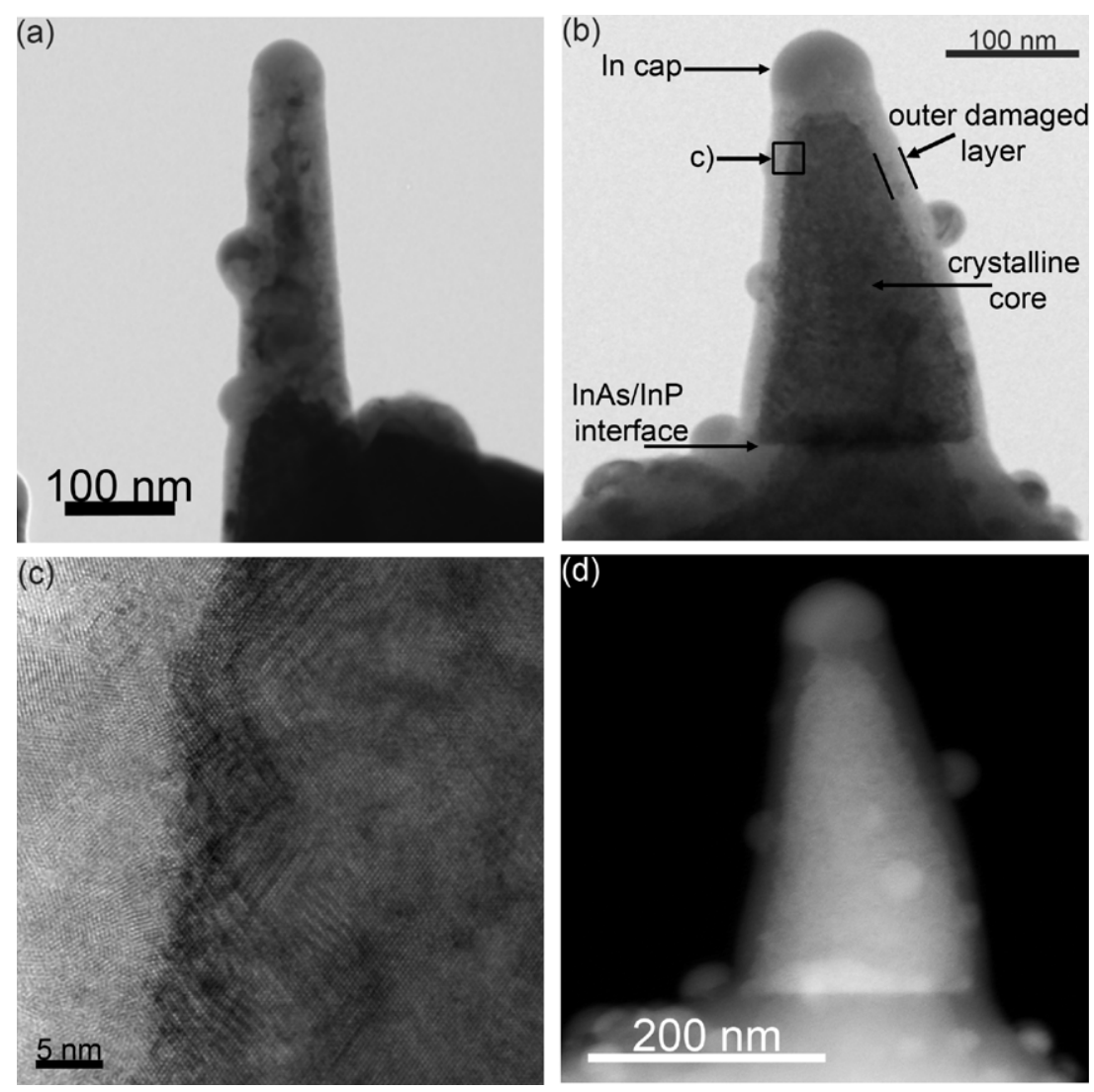

Figure 6. (a) shows a $\langle 110\rangle$ bright-field TEM image of an untemplated nanospike produced using an InAs/InP heterostructure and the identified optimum beam parameters for nanospike creation. (b) shows a $\langle 110\rangle$ bright-field TEM image of a templated nanospike on InAs/InP, with the different regions of the nanospike structure labeled. (c) is a high resolution TEM image taken at the area indicated in (b) showing the boundary between the outer ion damaged layer and the crystalline core of the spike. (d) is a HAADF STEM image of the nanospike pictured in (b) which more clearly shows the boundaries between nanospike features and the InAs/InP interface.

figure 5(d) shows the same area following 9000 passes of a 7.45 pA FIB beam using a 100 ns dwell time, resulting in the creation of one large primary nanospike at each mesa location. By pre-patterning and FIB irradiating InAs/InP it was found that nanospike formation can be reliably confined to just the center of those areas defined by the original mesa template, and that the number of nanospikes per mesa center can be varied from approximately 1-3 depending on prepatterning beam parameters and mesa size. Recessed areas in a FIB pre-pattern define the locations at which the InAs/InP interface and underlying InP substrate will first be exposed, and because nanospikes will not form on InP this acts to limit nanospike formation to the raised mesa regions of InAs. As the mesa edges are rapidly milled away the InAs/InP interface is driven toward the center of the mesa, acting to direct nanospike formation to the center of each original mesa location. Secondary nanospike formation between the mesas is prevented in a similar manner. The pre-patterning process thins or completely removes the InAs film between the mesas, resulting in early exposure of the InP substrate at those locations and so prevents nanospike formation. These mechanisms for controlling nanospike location place an inherent limit on the size and types of pre-patterned features that may be used to produce accurate templating. If the mesas are made increasingly large they will cease to effectively control the movement of the In droplets and multiple spikes at random locations within the mesa area may form. Likewise, if the mesa size is reduced too much, rapid milling from the mesa edges and a very small area for possible nanospike formation act to limit nanospike creation and the templating effect. In this work the smallest square mesa size successfully used to create templated nanospikes was $400 \mathrm{~nm} \times 400 \mathrm{~nm}$.

\subsection{Nanospike structure}

High resolution TEM examination of nanospikes produced from an InAs/InP heterostructure without templating revealed that they are capped with an amorphous or partially crystalline metallic tip, possess an ion damaged outer layer that is amorphous with small regions of local crystallinity, and contain a crystalline core. The conditions of the spike cores range from highly damaged and only partially crystalline to single crystalline. A cross-sectional bright-field TEM image of an untemplated InAs/InP nanospike with an only partially crystalline core can be seen in figure 6(a). InAs/InP nanospikes produced by templating also contain a metallic tip and outer damaged layer, but were in all cases observed to possess a single crystalline core that maintained the crystallographic orientation of the underlying substrate. Figure 6(b) shows a bright-field image of a templated nanospike with these features 
labeled. The core contains a layer of crystalline defects at its interface with the outer damaged shell, as shown in figure 6(c). The damaged shell is due to ion irradiation and could likely be recrystallized by annealing under an arsenic overpressure $[19,20]$. The single crystal nanospike cores retain the original zinc blende structure of the starting film and are free of extended defects such as twin boundaries or stacking faults commonly observed to span the width of semiconductor nanowires produced by VLS growth [21]. The bases of the nanospikes were found to be at or below the InAs/InP interface, which remained sharp and free of defects. A HAADF STEM image of the same nanospike pictured in figure 6(b) is shown in figure 6(d). HAADF STEM imaging and STEM/EDS mapping revealed that the spike tip is In and that the outer damaged layer is InAs partially depleted of As. This is not unexpected, as a FIB will preferentially sputter As from InAs [12, 22].

\section{Conclusions}

This work has demonstrated a method for creating and templating high aspect ratio nanospikes from both homoepitaxial InAs and InAs/InP heterostructures using a FIB erosion method. Using the identified optimum FIB parameters nanospikes taller than $\sim 950 \mathrm{~nm}$ may be created from homoepitaxial InAs, and nanospikes up to $\sim 875 \mathrm{~nm}$ tall may be created from an InAs/InP heterostructure with a $500 \mathrm{~nm}$ thick InAs film. Nanospike formation is attributed to stationary In droplets on the film acting while local etch masks as the surrounding material is etched away. When nanospikes are produced using homoepitaxial InAs, In droplets constantly nucleate and become stationary, allowing nanospikes to continually form throughout the erosion process. For the InAs/InP heterostructure case, it was found that no new nanospikes form on exposed InP, and so the time over which nanospikes can form and grow is controlled by the local starting thickness of the InAs film. Additionally, the location of the InAs/InP interface affects the locations at which nanospikes form by limiting the areas where large In droplets can exist to produce nanospikes. By using FIB pre-patterning to template the starting morphology of the InAs film, it was found that the locations at which nanospikes form on homoepitaxial InAs can be partially controlled. However, as continuous nanospike formation and the erosion of the initial pattern proceeds, the templating effect is disrupted. On the other hand, patterning heteroepitaxial InAs/InP can be used to more reliably control nanospike placement. Because nanospikes will not form on regions of exposed InP, FIB pre-patterning acts to limit the available area over which nanospikes can form. As erosion proceeds, full exposure of the underlying
InP acts as an inhibitor for nanospike formation. Thus using an InAs/InP heterostructure with a known InAs film thickness provides an additional degree of control over where and when nanospikes may form. The method for the creation of III-V semiconductor nanospikes described in this work is a unique approach to the creation of high aspect ratio nanostructures which provides a compromise between the placement accuracy of serial fabrication techniques and the speed of other ion beam and bottom-up self-organization techniques.

\section{References}

[1] Hu J, Odom T W and Lieber C M 1999 Acc. Chem. Res. 32 435-45

[2] Gudiksen M S, Lauhon L J, Wang J, Smith D C and Lieber C M 2002 Nature 415 617-20

[3] Xia Y, Yang P, Wu Y, Mayers B, Gates B, Yin Y, Kim F and Yan H 2003 Adv. Mater. 15 353-89

[4] Li D, Wu Y, Kim P, Shi L, Yang P and Majumdar A 2003 Appl. Phys. Lett. 83 2934-5

[5] Li D, Wu Y, Fan R, Yang P and Majumdar A 2003 Appl. Phys. Lett. 83 3186-8

[6] Tanemura M, Aoyama S, Fujimoto Y and Okuyama F 1991 Nucl. Instrum. Methods Phys. Res. B 61 451-6

[7] Nozu M, Tanemura M and Okuyama F 1994 Surf. Sci. Lett. 304 L468-74

[8] Chini T K, Kato J, Tanemura M and Okuyama F 1995 Nucl. Instrum. Methods Phys. Res. B $95313-8$

[9] Yuba Y, Hazama S and Gamo K 2003 Nucl. Instrum. Methods Phys. Res. B 206 648-52

[10] Nerbo I S, Kildemo M, Le Roy S, Simonsen I, Sondergard E, Holt L and Walmsley J C 2008 Appl. Opt. 47 5130-9

[11] Le Roy S, Barthel E, Brun N, Lelarge A and Sondergard E 2009 J. Appl. Phys. 106094308

[12] Lugstein A, Weil M, Basnar B, Tomastik C and Bertagnolli E 2004 Nucl. Instrum. Methods Phys. Res. B 222 91-5

[13] Lugstein A, Basnar B, Brezna W, Weil M, Golka S and Bertagnolli E 2006 Nucl. Instrum. Methods Phys. Res. B 242 93-5

[14] Callegari V and Nellen P M 2007 Phys. Status Solidi a 204 1665-71

[15] Szot J, Hornsey R, Ohnishi T and Minagawa S 1992 J. Vac. Sci. Technol. B 10 575-9

[16] Grossklaus K A and Millunchick J M 2011 J. Appl. Phys. 109014319

[17] Bradley R M and Harper J M E 1988 J. Vac. Sci. Technol. A $62390-5$

[18] Le Roy S, Sondergard E, Nerbo I S, Kildemo M and Plapp M 2010 Phys. Rev. B 81161401

[19] Pearton S J, Von Neida A R, Brown J M, Short K T, Oster L J and Chakrabarti U K 1988 J. Appl. Phys. 64 629-36

[20] Wesch W 1992 Nucl. Instrum. Methods Phys. Res. B 68 342-54

[21] Glas F, Harmand J C and Patriarche G 2007 Phys. Rev. Lett. 99146101

[22] Lugstein A, Basnar B, Smoliner J, Bertagnolli E and Weil M 2004 J. Vac. Sci. Technol. B 22 2995-9 